\title{
Quantifying Nutritional Trade-Offs across Multidimensional Performance Landscapes
}

\author{
Juliano Morimoto $^{1, \star}$ and Mathieu Lihoreau ${ }^{2}$ \\ 1. Department of Biological Sciences, Macquarie University, Sydney, New South Wales 2109, Australia; 2. Research Center on Animal \\ Cognition, Center for Integrative Biology, CNRS, University Paul Sabatier, Toulouse, France
}

Submitted June 6, 2018; Accepted November 14, 2018; Electronically published April 4, 2019

Online enhancements: R code, supplemental material. Dryad data: https://dx.doi.org/10.5061/dryad.tp7519s.

\begin{abstract}
A BSTRACT: Animals make feeding decisions to simultaneously maximize fitness traits that often require different nutrients. Recent quantitative methods have been developed to characterize these nutritional trade-offs from performance landscapes on which traits are mapped on a nutrient space defined by two nutrients. This limitation constrains the broad applications of previous methods to more complex data, and a generalized framework is needed. Here, we build on previous methods and introduce a generalized vector-based approach - the vector of position approach - to study nutritional trade-offs in complex multidimensional spaces. The vector of position approach allows the estimate of performance variations across entire landscapes (peaks and valleys) and comparison of these variations between animals. Using landmark published data sets on life span and reproduction landscapes, we illustrate how our approach gives accurate quantifications of nutritional trade-offs in two- and three-dimensional spaces and can bring new insights into the underlying nutritional differences in trait expression between species. The vector of position approach provides a generalized framework for investigating nutritional differences in life-history trait expression within and between species, an essential step for the development of comparative research on the evolution of animal nutritional strategies.
\end{abstract}

Keywords: nutritional geometry, nutritional trade-off, performance landscapes, fitness.

\section{Introduction}

Nutrition underpins complex life-history traits that determine individual fitness and impact on evolutionary processes (Simpson and Raubenheimer 2012). Recent advances in nutritional ecology show how an animal's diet can differently influence the expression of key life-history traits, lead-

\footnotetext{
* Corresponding author; email: juliano.morimoto@mq.edu.au. ORCIDs: Morimoto, https://orcid.org/0000-0003-3561-1920.
}

Am. Nat. 2019. Vol. 193, pp. E168-E181. (C) 2019 by The University of Chicago. $0003-0147 / 2019 / 19306-58530 \$ 15.00$. All rights reserved. This work is licensed under a Creative Commons Attribution-NonCommercial 4.0 International License (CC BY-NC 4.0), which permits non-commercial reuse of the work with attribution. For commercial use, contact journalpermissions@press.uchicago.edu. DOI: $10.1086 / 701898$ ing animals to trade off between optimizing multiple traits simultaneously (e.g., Bunning et al. 2015, 2016; Morimoto and Wigby 2016; Rapkin et al. 2018). In insects, for instance, life span is typically enhanced on high-carbohydrate diets, whereas reproduction is maximized on high-protein diets (e.g., fruit flies [Lee et al. 2008; Fanson et al. 2009; Reddiex et al. 2013; Jensen et al. 2015; Semaniuk et al. 2018], crickets [Maklakov et al. 2008]). Immunity and reproduction also display complex species-specific differences in nutritional requirements (e.g., moths [Cotter et al. 2011], fruit flies [Ponton et al. 2015], crickets [Rapkin et al. 2018]; reviewed in Ponton et al. 2011; Schwenke et al. 2016). Even traits related to different stages of reproduction can have specific nutritional requirements that are not attainable simultaneously (fruit flies [Reddiex et al. 2013; Jensen et al. 2015; Morimoto and Wigby 2016], cockroaches [Bunning et al. 2015], beetles [House et al. 2016]). Measuring these nutritional trade-offs is challenging because it requires the separation of the potential confounding effects of energy intake and nutrient balance in determining the expression of traits. Only by controlling for these confounding effects is it possible to identify the specific food components required for the expression of one trait over another (Stearns 1989; Roff 2002; Hunt et al. 2004).

In recent years, concepts of nutritional geometry (also known as the geometric framework for nutrition; Raubenheimer and Simpson 1993; Simpson and Raubenheimer 1993, 2012) have been increasingly used to experimentally generate performance landscapes on which the expression of a fitness trait can be mapped onto a nutrient space defined by the intake of two or more nutrients, a powerful approach to disentangle the effects of nutrient and energy intake on the optimal expression of traits (e.g., Lee et al. 2008; Maklakov et al. 2008). Nutritional geometry has provided key insights into the nutritional factors underpinning a wide variety of physiological and behavioral phenomena across taxonomic groups, feeding guilds, and ecological contexts (e.g., Trumper and Simpson 1993; Simpson et al. 2010; Lihoreau et al. 2015; Raubenheimer and Simpson 2018), including in 
human societies (Gosby et al. 2014). In most cases, nutritional trade-off analyses were performed using simple experimental designs (i.e., two-dimensional nutrient spaces) and based on the visual inspection of peaks in performance landscapes (Simpson and Raubenheimer 2012). However, with the fast development of nutritional geometry studies in ecology and evolution, powerful analyses are needed in order to accurately assess nutritional trade-offs in complex nutrient spaces (i.e., high-dimensional data) and compare trade-offs within and between species. For instance, studies have begun to investigate the effects of individual amino acids (rather than full proteins) on the expression of fitness traits (e.g., Grandison et al. 2009; Arganda et al. 2017; Piper et al. 2017). This systematic assessment of protein components (and eventually other nutrients) results in nutritional geometry landscapes with dozens of dimensions, for which visual inspection is impossible. Furthermore, applications of nutritional geometry in biological and health-related research have raised the need for accurately quantifying and comparing patterns of variations across performance landscapes, such as, for instance, regions where performances reach their highest (i.e., "peaks") but also their lowest (i.e., "valleys") points. The possibility of identifying peaks and valleys is crucial for estimating the best and worst diets involved in the onset, progression, and/or treatment of diseases (e.g., Gosby et al. 2014; Solon-Biet et al. 2014, 2015; Simpson et al. 2017a, 2017b). Developing a standard quantitative method for analyzing these nutritional data has therefore become a key issue for comparative research and for investigating the role of nutrition in the evolution of physiology and behavior (Lihoreau et al. 2015).

Bunning et al. (2015) and Rapkin et al. (2018) recently proposed a method for calculating the angle and distance between the peaks of fitness traits in two-dimensional nutrient spaces (henceforth referred as the angular method; see the description in "Material and Methods" and fig. $1 A-1 G$ ). This method is based on the representation of performance landscapes as vectors of slopes and has been applied to measure nutritional trade-offs between immunity and reproduction as well as between different reproductive traits within species (Bunning et al. 2015, 2016; Rapkin et al. 2016, 2018). Using vectors of slopes permits estimates of the strength of nutritional trade-offs that are prohibitively higher than the upper limit allowed in nutritional geometry studies, potentially resulting in mismatching expectations of the real strength of the nutritional trade-offs in performance landscapes. While this is a workable solution for simple nutritional designs, the potential risk of misinterpretation of performance landscapes may rapidly become more significant as the analyses become more complex-for instance, in the context of comparative studies, when multiple performance landscapes must be compared, or in studies using more complex (highdimension) nutritional spaces. Additionally, this method uses regression models to estimate nutritional trade-offs, which does not allow for the identification and comparison of other key features of the performance landscapes, such as valleys, and therefore overlooks important information about how performances vary across the entire landscape.

To address these limitations, we build on the angular method and introduce a generalized vector-based approach to accurately measure and compare nutritional trade-offs in multidimensional performance landscapes while allowing for analyses of novel landscape parameters: the vector of position approach. First, we show that this novel approach provides an accurate measure of the angle between the peaks and valleys of the traits and an in-depth description of the nutritional trade-offs using a landmark data set on life spanreproduction trade-offs in Drosophila (Lee et al. 2008). Second, we use simulated data to illustrate how the vector of position approach can be generalized to more complex highdimension nutrient spaces, allowing for the comparison of the trade-offs in studies with three or more nutrients. Third, we show how our analyses can be applied for between-species comparisons, using published data sets on life span-reproduction trade-offs in two fruit fly species (Lee et al. 2008; Fanson et al. 2009).

\section{Material and Methods \\ Overview of the Two Methods}

The Angular Method. The angular method uses vectors to describe the relationship between nutrient intake and the expression of traits and measures the angle between the vectors to infer the presence and strength of nutritional trade-offs (Bunning et al. 2015). To obtain the coordinates of the vector, the first step is to fit a linear regression of the trait onto the intake of each nutrient of interest, forcing the intercept to zero. The slope estimates for the intake of each nutrient onto the performance trait (e.g., life span) become the coordinates of the vector. For example, if we consider two nutrients - for example, protein $(P)$ and carbohydrate $(C)$ - we have a regression,

$$
\text { trait }_{i} \sim \beta_{P, i} P+\beta_{C, i} C-1,
$$

where the slopes $\beta_{P, i}$ and $\beta_{C, i}$ the linear slopes of $P$ and $C$ onto the performance trait $i$-are used as the components of a two-dimensional vector, $\vec{a}_{i}$,

$$
\vec{a}_{i}=\left(\beta_{P, i} \quad \beta_{C, i}\right) .
$$

In a simple example with two traits - trait $\mathrm{A}$ and trait $\mathrm{B}$ with peaks in different regions of the nutrient space (fig. $1 \mathrm{~A}$, $1 B$ ), the vectors of slopes can be defined as follows (fig. $1 C$ ):

$$
\begin{aligned}
& \vec{a}_{\mathrm{A}}=\left(\begin{array}{ll}
\beta_{P, \mathrm{~A}} & \beta_{C, \mathrm{~A}}
\end{array}\right), \\
& \vec{a}_{\mathrm{B}}=\left(\begin{array}{ll}
\beta_{P, \mathrm{~B}} & \beta_{C, \mathrm{~B}}
\end{array}\right) .
\end{aligned}
$$


A

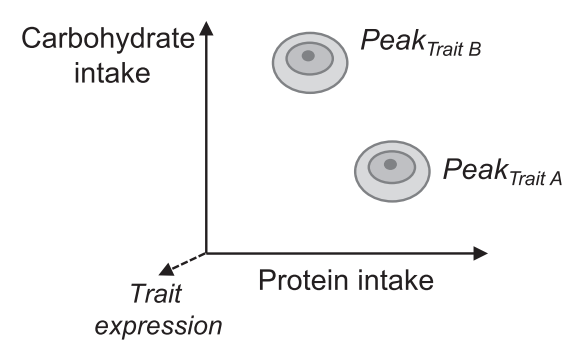

C

Angular Method

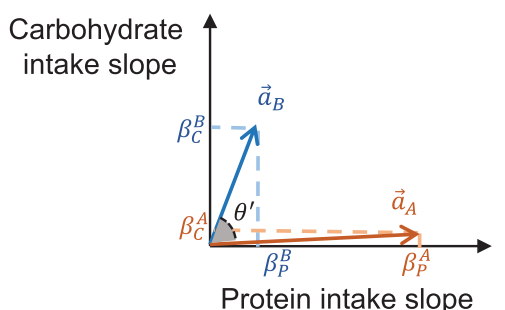

E

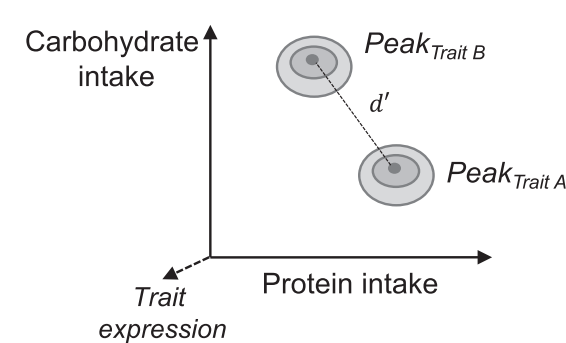

G

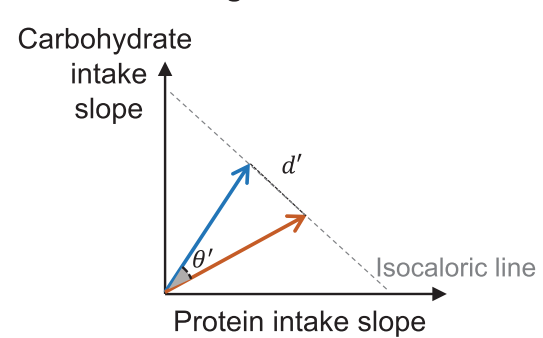

B

Angular Method

$\vec{a}_{i}=\left(\begin{array}{ll}\beta_{\mathrm{P}, i} & \beta_{\mathrm{C}, i}\end{array}\right)$

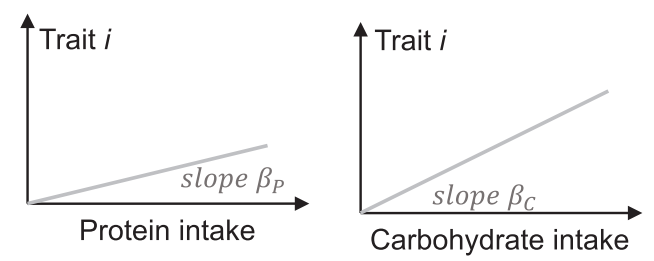

D

Angular Method

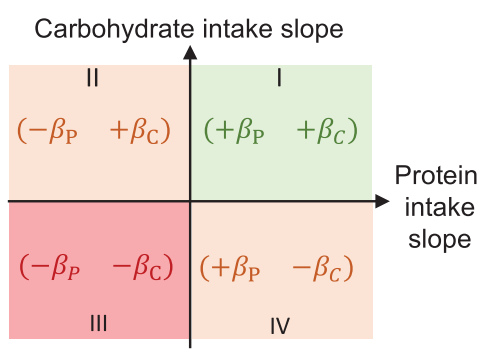

F

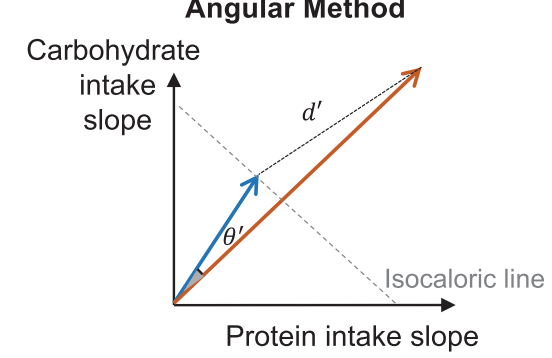

H Vector of Position Approach

Carbohydrate

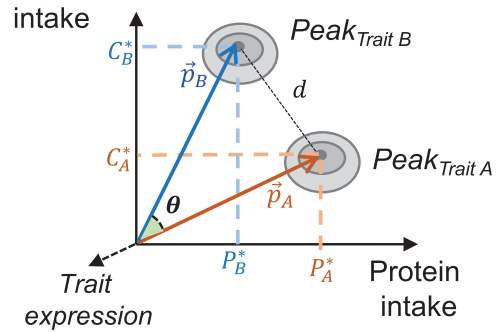

Figure 1: Overview of the angular method and the vector of position approach. $A$, Hypothetical performance landscape with peaks of traits $\mathrm{A}$ and $\mathrm{B}$ (gray regions). $B$, Linear slopes of protein and carbohydrate intake onto the trait $i$ that composes the vector $\vec{a}_{i}$. $C$, Angle $\theta^{\prime}$ that separates the vectors $\vec{a}_{\mathrm{A}}$ and $\vec{a}_{\mathrm{B}}$. D. Information on the slopes within slope vectors $\vec{a}_{i}$. Note that the direction of vectors $\vec{a}_{i}$ in the Cartesian plane contains information about the sign of the slopes of protein and carbohydrate intake onto the trait $i$. E, Euclidean distance $d^{\prime}$ between the peaks of traits A and B. F, Schematic representation of the relationship between $\theta^{\prime}$ and $d^{\prime}$ for nutritional peaks in different isocaloric lines. $G$, Schematic representation of the relationship between $\theta^{\prime}$ and $d^{\prime}$ for nutritional peaks in the same isocaloric line. $H$, Position vectors $\vec{p}_{i}$ that describe the relationship between the peaks of traits $\mathrm{A}$ and $\mathrm{B}$ in the performance landscape. 
Each of these vectors of slope can be considered as a summarized description of the relationship between the intake of $P$ and $C$ and the expression of traits $A$ and $\mathrm{B}$, respectively. It is then possible to compare these vectors by calculating the angle $\theta^{\prime}$ formed between the $\vec{a}_{\mathrm{A}}$ and $\vec{a}_{\mathrm{B}}$ vectors, as shown in figure $1 C$, as

$$
\theta^{\prime}=\cos ^{-1}\left(\frac{\vec{a}_{\mathrm{A}} \cdot \vec{a}_{\mathrm{B}}}{\left\|\vec{a}_{\mathrm{A}}\right\|\left\|\vec{a}_{\mathrm{B}}\right\|}\right),
$$

where $\left\|\vec{a}_{A}\right\|$ and $\left\|\vec{a}_{B}\right\|$ are the length of the vectors of slope $\vec{a}_{\mathrm{A}}$ and $\vec{a}_{\mathrm{B}}$, respectively. (Note: In the original proposition of the angular method, the angle between these vectors of slopes was called $\theta$ instead of $\theta^{\prime}$; Rapkin et al. 2018.)

Because $\theta^{\prime}$ is a measure of difference (i.e., angular distance) between the vectors of slope associated with performance traits, $\theta^{\prime}$ has been used as a measure of the strength of the nutritional trade-off underlying the expression of these traits (Rapkin et al. 2018). Note that $\theta^{\prime}$ can assume values between $0^{\circ}$ and $180^{\circ}$. The closer the value of $\theta^{\prime}$ is to $0^{\circ}$, the more aligned the peaks in the nutrient space are expected to be; the closer the value of $\theta^{\prime}$ is to $180^{\circ}$, the more distant the peaks are located in relation to each other (Rapkin et al. 2018). Therefore, larger values of the angle $\theta^{\prime}$ indicate stronger nutritional trade-offs on the expression of two performance traits. Because the coordinates of the vectors of slope $\vec{a}_{i}$ are obtained by the linear slopes $\beta_{P}$ and $\beta_{C}$ onto the performance trait (fig. $1 A-1 C$ ), the angle $\theta^{\prime}$ measures how different the slopes of $P$ and $C$ are between each of the performance traits. This means that the position where a given $\vec{a}_{i}$ vector exists in the Cartesian plane yields information about the sign of the slope of the nutrient on the expression of the performance trait. For example, if $\vec{a}_{i}$ lies on the second quadrant (upper left-hand region of the Cartesian plane; II in fig. 1D), the slope of $C$ intake is positive (i.e., eating more $C$ increases the performance trait), while the slope of the $P$ intake is negative (i.e., eating more $P$ decreases the performance trait; fig. $1 D$ ). This property will be explored to estimate the strength of the nutritional trade-offs using the vector of position approach (see "Results").

It is important to note that the estimates of the slopes $\beta_{P, i}$ and $\beta_{C, i}$ can assume any value in the real range (i.e., positive, negative, or zero values), meaning that the vectors $\vec{a}_{i}=$ $\left(\beta_{P, i} \beta_{C, i}\right)$ can exist in any of the four quadrants of the Cartesian plane (see fig. $1 D$ ). However, in nutritional geometry, the nutrient space, which is the region in which the performance landscape exists, is defined only within the range of positive numbers (Simpson and Raubenheimer 2012). In other words, the nutritional space exists only in the first quadrant of the plane, and it is impossible for the performance landscape to exist outside the first quadrant of the Cartesian plane because there is no "negative" intake of any nutrient. Therefore, vectors with negative coordinates are undefined from the point of view of the nutrient space, and any measure of distance between vectors with negative components cannot represent the true angle between the peaks observed in the performance landscapes.

In addition to $\theta^{\prime}$, Rapkin et al. (2018) extended the angular method to include estimates of the Euclidean distances $d^{\prime}$ between the peaks of two traits in the nutrient spaces (fig. 1E). The Euclidean distance measures the shortest distance between one point and another in the nutritional landscapes (e.g., the distance between two peaks; see fig. 1E) and can be calculated as

$$
d^{\prime}=\sqrt{\left(P_{\mathrm{A}}^{*}-P_{\mathrm{B}}^{*}\right)^{2}+\left(C_{\mathrm{A}}^{*}-C_{\mathrm{B}}^{*}\right)^{2}} \text { units }
$$

or according to geometrical laws (Heath 1956):

$$
d^{\prime}=\sqrt{\vec{a}_{\mathrm{A}}^{2}+\vec{a}_{\mathrm{B}}^{2}-2 \vec{a}_{\mathrm{A}} \cdot \vec{a}_{\mathrm{B}} \cos \left(\theta^{\prime}\right)} \text { units, }
$$

where $P_{i}^{*}$ and $C_{i}^{*}$ are the $P$ and $C$ coordinates of the peak in the performance landscapes of trait $i, \vec{a}_{\mathrm{A}}$ and $\vec{a}_{\mathrm{B}}$, and the slope vectors of traits $\mathrm{A}$ and $\mathrm{B}$ (as described above) and $\theta^{\prime}$ is the angle between the vectors of slope of traits $A$ and $B$. Units are given in milligrams, grams, or any other unit used when measuring nutrient intake. Rapkin et al. (2018) suggested that if the peaks are located in different isocaloric lines (i.e., lines with slope -1 ) in the nutrient space, $d^{\prime}$ becomes a more reliable measure of the nutritional trade-off than $\theta^{\prime}$ because $\theta^{\prime}$ can underestimate the true distance between the peaks (Rapkin et al. 2018; fig. 1F). However, if the peaks lie in the same isocaloric lines, large $\theta^{\prime}$ are associated with large $d$ and vice versa (fig. $1 G$ ). We discuss the validity of equation (5b) in the angular method in the next section (see below).

The Vector of Position Approach. The vector of position approach is an extension of the angular method that builds on the representation of performance landscapes through vectors while removing potential confusions regarding the interpretations of the angle $\theta^{\prime}$ (see above) by using vectors of positions $\vec{p}_{i}$ instead of vectors of slopes. Here, the coordinates of the vector are the point coordinates of the peak in the performance landscape (see, e.g., the OptimaRegion package in R [del Castillo et al. 2016] for this purpose). In the vector of position approach, the coordinates of the vector of position $\vec{p}_{i}$ can also be the point coordinates of the valleys in the performance landscape, meaning that this new method allows for the comparisons of nutritional trade-offs that maximize and/or minimize performance landscapes (for an example of this application, see "Results").

The point coordinates of the peak in performance trait expression are $P^{*}$ and $C^{*}$, whereby $P^{*}$ and $C^{*}$ are the $P$ and $C$ intake, respectively, that maximize the trait. The vector of position $\vec{p}_{i}$ can thus be represented as 


$$
\vec{p}_{i}=\left(\begin{array}{ll}
P_{i}^{*} & C_{i}^{*}
\end{array}\right),
$$

where $i$ is the performance trait. Using the example above, the position vectors for traits $\mathrm{A}$ and $\mathrm{B}$ are as follows (see fig. $1 \mathrm{H}$ ):

$$
\begin{aligned}
& \vec{p}_{\mathrm{A}}=\left(\begin{array}{ll}
P_{\mathrm{A}}^{*} & C_{\mathrm{A}}^{*}
\end{array}\right), \\
& \vec{p}_{\mathrm{B}}=\left(\begin{array}{ll}
P_{\mathrm{B}}^{*} & C_{\mathrm{B}}^{*}
\end{array}\right) .
\end{aligned}
$$

Having defined the coordinates of the position vectors, it is possible to calculate the observed degree of separation - the angle $\theta$-between the vectors $\vec{p}_{\mathrm{A}}$ and $\vec{p}_{\mathrm{B}}($ fig. $1 H$ ) as

$$
\theta=\cos ^{-1}\left(\frac{\vec{p}_{\mathrm{A}} \cdot \vec{p}_{\mathrm{B}}}{\left\|\vec{p}_{\mathrm{A}}\right\|\left\|\vec{p}_{\mathrm{B}}\right\|}\right) .
$$

Using the vectors of positions guarantees that $\vec{p}_{\mathrm{A}}$ and $\vec{p}_{\mathrm{B}}$ always lie in the defined region of the performance landscape (i.e., the first quadrant of the Cartesian plane). An angle $\theta=$ $90^{\circ}$ would represent trait performance peaks that are located at the exact opposite ends of the nutrient space but still within the first quadrant. This is important when the intake of a nutrient is inversely correlated with the expression of the trait being analyzed since in these cases the slope of the nutrient onto the performance trait expression $\beta_{i}$ is negative and the vector of slope $\vec{a}$ (as considered in the angular method) would lie in the second, third, or fourth quadrant of the Cartesian plane, which is outside the defined region of the nutrient space (as described previously).

Once $\theta$ is calculated, we can calculate the Euclidean distance $d$ between the peaks of two traits as follows (see fig. $1 H$ ):

$$
d=\sqrt{\vec{p}_{\mathrm{A}}^{2}+\vec{p}_{\mathrm{B}}^{2}-2 \vec{p}_{\mathrm{A}} \cdot \vec{p}_{\mathrm{B}} \cos (\theta)} .
$$

The law of cosines (Heath 1956), which calculates the length of the side of a triangle from the value of two sides and the cosine of the angle between them, formalizes the relationship between $\theta$ and $d$. Note, however, that the law of cosines cannot be used to calculate $d^{\prime}$ using the $\theta^{\prime}$ (see eq. [5b]) because $\theta^{\prime}$ does not measure the true angle between the peaks in the performance landscapes (see "Results").

Data and Statistical Analyses. The data for the Drosophila melanogaster study were obtained from Lee et al. (2008). The data for the Queensland fruit fly Bactrocera tryoni study were obtained from Fanson et al. (2009). In brief, both studies used nutritional geometry to investigate the effects of protein and carbohydrate intake on life span, lifetime reproduction (total number of eggs laid), and reproductive rate (RR; number of eggs laid per day; for details, see "Supplementary Methods," available online in the supplemental material).

All analyses were performed in R version 3.4.0 ( $\mathrm{R}$ Development Core Team 2017). Step-by-step R scripts for the data analyses are provided in supplemental materials. ${ }^{1}$ Performance landscapes were plotted using the Tps() function of the fields package (Nychka et al. 2017). To obtain the point estimates for peaks and valleys in the performance landscapes on the absolute scale, we extracted the coordinates of the maximum and minimum fitted values of the function Tps, respectively. To obtain the slopes of $P$ and $C$ intake on life span, lifetime reproductive success (LRS), and RR, we used the function MCMCglmm() from the MCMCglmm package (Hadfield 2010), with uninformative prior $n u=0.2$, expected covariances $V=1,400,000$ iterations, a burn-in of 2,000, and a thinning parameter of 25 (as in Bunning et al. 2015). No autocorrelation was detected in the model according to diagnostic trace plots. Model convergence and robustness to different prior parameters were tested by varying both $V$ and $n u$. We observed a low standard deviation of the posterior mean, which corroborates the convergence and robustness of the models to different priors (see table S1, available online). As in studies using the angular method (Bunning et al. 2015; Rapkin et al. 2018), 95\% credible intervals (CIs) were calculated from the posterior distribution. For the comparison between the two species, we standardized the $P$ and $C$ intake as well as the trait values by mean subtraction, as described in "Results" (eqq. [13], [14]). To estimate the optimum region in the standardized performance landscapes, we used a support-vector machine regression model with the caret package (Kuhn 2017), from which we could select the coordinates of the simulated $P$ and $C$ intake that resulted in the predictions that matched with the optimum region in the performance landscapes (for details, see "Supplementary Methods"). All data sets used in this study have been deposited in the Dryad Digital Repository (https://dx.doi.org/10 .5061/dryad.tp7519s; Morimoto and Lihoreau 2019).

\section{Results}

The Vector of Position Approach Estimates the True Degree of Separation between Peaks in the Performance Landscape

To illustrate the advantage of the vector of position approach relative to the angular method, we analyzed and compared data from Lee et al. (2008) with both methods. This study explored nutritional trade-offs between life span, LRS, and RR in Drosophila melanogaster. Visual inspection of the performance landscapes indicates that life span is maximized in diets with a low $P: C$ ratio of $\sim 1: 16$ and that LRS is maximized at a $P: C$ ratio of $\sim 1: 4$, whereas $\mathrm{RR}$ is maximized at a $P: C$ ratio of $\sim 1: 2$ (fig. 2 ).

Using the angular method, we estimated the angle $\theta^{\prime}$ between the vectors of slope $\vec{a}_{\mathrm{RR}}, \vec{a}_{\mathrm{LRS}}$, and $\vec{a}_{\text {life span }}$. The

1. Code that appears in The American Naturalist is provided as a convenience to readers. It has not necessarily been tested as part of peer review. 
A

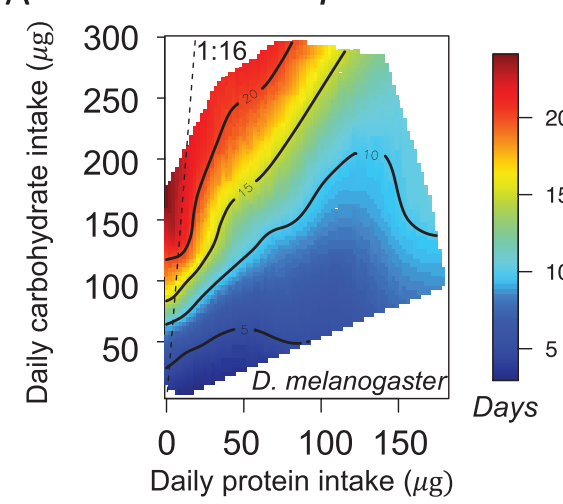

C

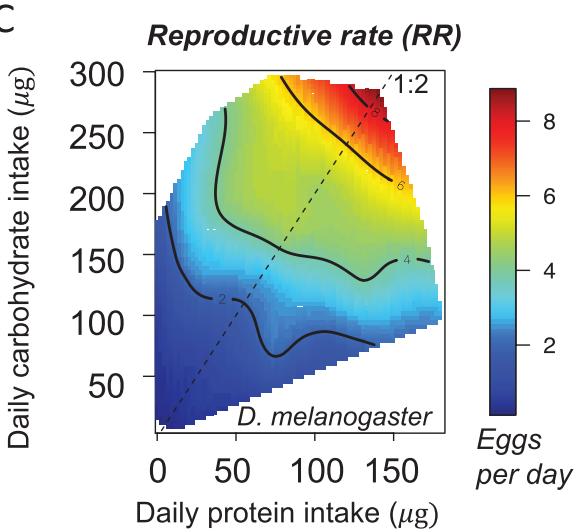

E Vector Projections

Carbohydrate

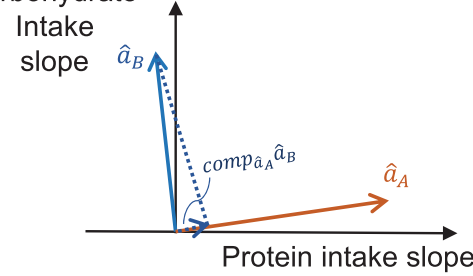

B

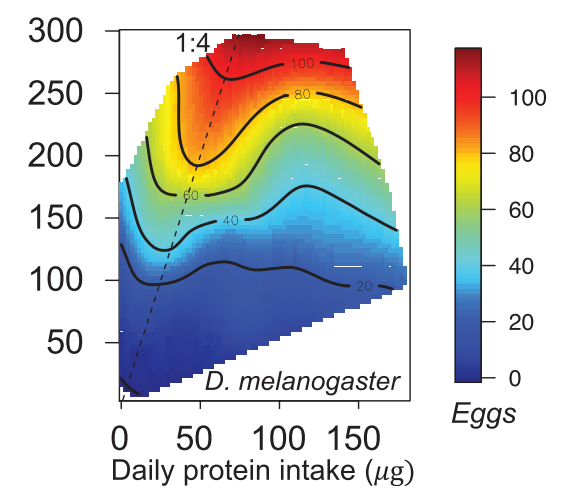

D

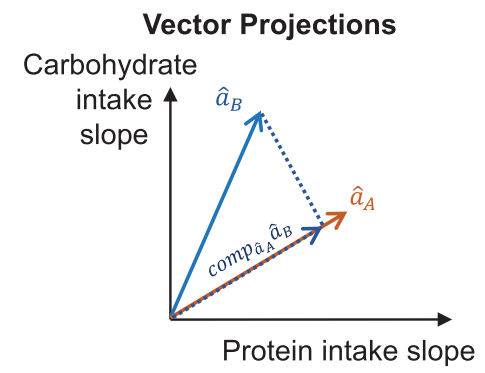

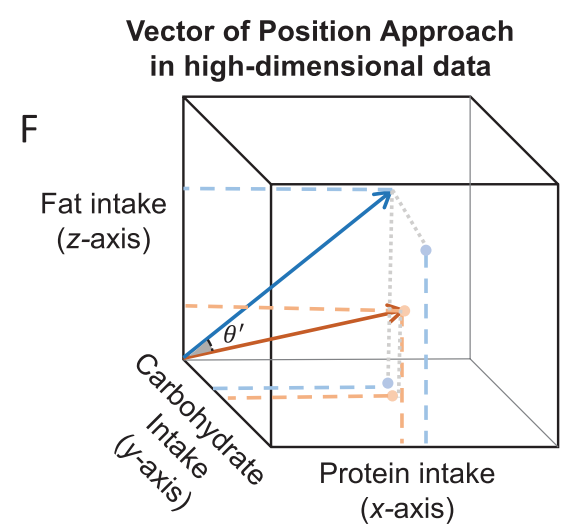

Figure 2: Performance landscapes and vector projections. $A-C$, Performance landscapes of life span (A), lifetime reproductive success (B), and reproductive rate $(C)$ of Drosophila melanogaster females (data from Lee et al. 2008). $D-E$, Vector projection to calculate the component of the unit vector $\vec{a}_{\mathrm{B}}$ onto the unit vector $\vec{a}_{\mathrm{A}}$ (i.e., comp $\hat{a}_{\mathrm{A}} \hat{a}_{\mathrm{B}}$ ). Unit vectors are standardized vectors of the same direction of the original vector of slopes but with lengths equal to 1. D, Example of a large component of $\vec{a}_{\mathrm{B}}$ into $\vec{a}_{\mathrm{A}}$. E. Example of a small component of $\vec{a}_{\mathrm{B}}$ into $\vec{a}_{\mathrm{A}}$. F, Schematic representation of a three-dimensional vector in a synthetic performance landscape of protein, carbohydrate, and fat intake using the vector of position approach. Data for the performance landscapes are available in the Dryad Digital Repository (https://dx.doi.org/10.5061 /dryad.tp7519s; Morimoto and Lihoreau 2019).

strength of the nutritional trade-off was high between life span and RR and between RR and LRS and was low between life span and LRS (table 1). The estimate of the distance $d^{\prime}$ was $294.89 \mathrm{mg}$ between life span and RR, $155.66 \mathrm{mg}$ between life span and LRS, and $261.91 \mathrm{mg}$ between LRS and RR.
Using the vector of position approach, we determined the coordinates of the vectors of position $\vec{p}_{i}$ as:

$$
\begin{aligned}
& \vec{p}_{\text {life span: }}\langle 0,176.717\rangle, \quad \vec{p}_{\text {LRS: }}\langle 75.001,299.174\rangle, \\
& \vec{p}_{\text {RR: }}\langle 141.198,284.488\rangle,
\end{aligned}
$$


Table 1: Matrix of estimates of the angles between vectors of positions $(\theta)$ and slopes $\left(\theta^{\prime}\right)$

\begin{tabular}{|c|c|c|c|}
\hline & Life span & LRS & $\mathrm{RR}$ \\
\hline Life span & & $\theta^{\prime}=15.082(12.022-18.123)$ & $\theta^{\prime}=63.210(59.876-66.438)$ \\
\hline LRS & $\theta=14.073(10.857-19.889)$ & & $\theta^{\prime}=48.112(43.945-52.149)$ \\
\hline $\mathrm{RR}$ & $\theta=26.390(25.471-27.385)$ & $\theta=12.322(7.496-14.613)$ & $\ldots$ \\
\hline
\end{tabular}

Note: Shown are nutritional trade-offs between life span and reproduction in Drosophila melanogaster (data from Lee et al. 2008) as calculated using the angular method $\left(\theta^{\prime}\right.$; above the diagonal of the matrix) and the vector of position approach $(\theta$; below the diagonal of the matrix). Estimates (95\% credible intervals) are given in degrees. LRS $=$ lifetime reproductive success; RR $=$ reproductive rate.

which corresponds to a $P: C$ ratio of $\sim 1: 16,1: 4$, and $1: 2$, as expected from visual inspection in Lee et al. (2008; fig. $2 A-$ $2 C)$. We then calculated the angles $\theta$ that separated the vectors $\vec{p}_{i}$ and found that the strength of the nutritional tradeoff was relatively high between life span and RR and low between life span and LRS and between RR and LRS (table 1). These estimates of the angles $\theta$ were confirmed using values of a standard table for $\theta$ based on the most commonly used $P: C$ ratios in nutritional geometry studies in Drosophila (see table S2).

We then calculated the Euclidean distance $d$ between the peaks of expression of traits according to $\vec{p}_{i}$ and $\theta$. As expected, the estimates of $d$ were longer between $\vec{p}_{\text {life span }}$ and $\vec{p}_{\mathrm{RR}}(d=205.02 \mathrm{mg})$ than between $\vec{p}_{\text {life span }}$ and $\vec{p}_{\mathrm{LRS}}(d=$ $154.25 \mathrm{mg})$ and between $\vec{p}_{\mathrm{RR}}$ and $\vec{p}_{\mathrm{LRS}}(d=94.90 \mathrm{mg})$, indicating a larger separation between the peaks of life span and RR (table 1). Together, these results confirmed the strong nutritional trade-offs among life span, LRS, and RR observed by Lee et al. (2008) and, more recently, by Reddiex et al. (2013) and Jensen et al. (2015).

The comparison between the two methods shows that the vector-based approach estimates the true degree of separation between peaks in the performance landscape where the angular method can lead to inaccurate results and misinterpretation. In this example, we found a twofold overestimation of the angle separating the peaks of life span and RR when calculating $\theta^{\prime}$ compared with $\theta$ and a fourfold overestimation of the angle separating the peaks of LRS and RR. Such overestimation of the distance between peaks using the angular method led to an erroneous interpretation of the $P: C$ ratio diets on which the peaks are maximized. A separation of $63.21^{\circ}$ given for the vectors of life span and RR is equivalent to a separation between $P: C$ ratios of $2: 1$ (high protein) and $0: 1$ (pure sugar), which are considerably more distant in the performance landscape than the true $P: C$ ratios of $1: 16$ to $1: 2$ that maximized life span and RRs (for angles between commonly used $P$ : $C$ ratios, see table S2). Moreover, the angular method suggested that there was a stronger nutritional trade-off between LRS and RR than between life span and LRS (see table 1). This misinterpretation was corrected by the vector of position approach, which revealed that the nutritional trade-off between LRS and RR is likely weak due to the proximity of the $P: C$ ratios maximizing these traits (i.e.,
$P: C$ ratios of $1: 4$ and $1: 2$, respectively). Furthermore, the inaccuracy in the estimation of the Euclidean distance between peaks was of $\sim 30.4 \%$ for LS and RR, $\sim 1 \%$ for LS and LRS, and $64.7 \%$ for LRS and RR, which likely contributed to the misinterpretation of the strength of the nutritional tradeoffs between LRS and RR described previously.

\section{The Vector of Position Approach Can Be Used to Estimate} Valleys in the Performance Landscape

Performance landscapes can yield much more information about the nutritional biology of animals than just peaks of trait expression. For instance, valleys (regions where the performances reach their minimum) can help identify the worst diets for the animals. The vector of position approach makes it possible to estimate such valleys, in contrast to the angular method, which uses regression models to estimate the coefficients of the vectors of slope. To exemplify this novel application, we calculated valleys and the separation between them in the performance landscapes of life span, LRS, and RR in the D. melanogaster data set (Lee et al. 2008; see table 2). The angle between the $P: C$ ratios of the valleys was estimated as $\theta_{\text {life span-LRS }}=23.880^{\circ}\left(95 \% \mathrm{CI}: 20.840^{\circ}-\right.$ $\left.27.840^{\circ}\right)$ for life span and LRS, $\theta_{\mathrm{LRS}-\mathrm{RR}}=0.000^{\circ}(95 \% \mathrm{CI}$ : $0.318^{\circ}-0.385^{\circ}$ ) for LRS and RR, and $\theta_{\text {life span-RR }}=23.880^{\circ}$ $\left(95 \% \mathrm{CI}: 20.521^{\circ}-28.200^{\circ}\right)$ for life span and RR. These results show greater separation between life span and LRS and between life span and RR than between LRS and RR. Interestingly, the valley of life span was estimated in the region where $\mathrm{RR}$ is maximized $(P: C$ ratio of $\sim 1: 2)$, suggesting

Table 2: Protein-to-carbohydrate $(P: C)$ ratios of the valleys in performance landscapes

\begin{tabular}{lc}
\hline Life-history trait & Estimate $(95 \% \mathrm{CI})$ \\
\hline Life span & $1: 1.87(1: 1.57-1: 2.17)$ \\
LRS & $1: 13.64(1: 12.41-1: 14.86)$ \\
RR & $1: 13.64(1: 13.55-1: 13.72)$ \\
\hline
\end{tabular}

Note: Shown are estimated $P: C$ ratios of the valleys in the landscapes for life span, lifetime reproductive success (LRS), and reproductive rate (RR) as calculated by the vector of position approach (data from Lee et al. 2008). Data are estimates (95\% credible intervals [CIs]). 
that these life-history traits have the strongest nutritional trade-off among the traits measured in the study.

\section{Vector Projections Can Be Used to Extract Information from Vectors of Slope}

The angle $\theta^{\prime}$ between vectors of slope contains important information about the relationship between $P$ or $C$ intake (or both) and the expression of the performance trait, as it reveals whether this relationship is positive, negative, or zero. Using the concepts of vector projection, the vector of slopes $\vec{a}_{i}$ can be used to quantify the influence of each nutrient on the expression of performance traits. For instance, consider a unit vector $\hat{a}_{i}$, a standardized vector with the same direction as the vector $\vec{a}_{i}$ but with length 1 , obtained by dividing the vector by its length:

$$
\hat{a}_{i}=\frac{\vec{a}_{i}}{\left\|\vec{a}_{i}\right\|} .
$$

Using unit vectors, we can calculate the scalar projection (the "scaling factor") of the unit vector $\hat{a}_{\mathrm{B}}$ onto the unit vector $\hat{a}_{\mathrm{A}}$ (fig. $2 D, 2 E$ ) as

$$
\operatorname{comp}_{\hat{a}_{\mathrm{A}}} \hat{a}_{\mathrm{B}}=\left(\frac{\hat{a}_{\mathrm{A}} \cdot \hat{a}_{\mathrm{B}}}{\left\|\hat{a}_{\mathrm{A}}\right\|}\right) .
$$

The scalar projection comp $\hat{a}_{\mathrm{A}} \hat{a}_{\mathrm{B}}$ measures how much of the vector $\hat{a}_{\mathrm{B}}$ is in the same direction as the vector $\hat{a}_{\mathrm{A}}$ (hence the saying "component of" $\hat{a}_{\mathrm{B}}$ onto $\hat{a}_{\mathrm{A}}$ ). Large values of the scalar projection comp $\hat{a}_{\mathrm{A}} \hat{a}_{\mathrm{B}}$ indicate that $\hat{a}_{\mathrm{B}}$ shares a large component in the direction of $\hat{a}_{\mathrm{A}}$, and therefore there exists only a weak potential for a nutritional trade-off between traits (e.g., fig. $2 D$ ). Conversely, small values of the scalar projection $\operatorname{comp}_{\hat{a}} \mathrm{~A} \hat{a}_{\mathrm{B}}$ indicate that $\hat{a}_{\mathrm{B}}$ shares only a small component in the direction of $\hat{a}_{\mathrm{A}}$, and therefore there exists a strong potential for a nutritional trade-off between traits $A$ and $B$ (e.g., fig. 2E).

We applied the vector of position approach to calculate the scalar projection of the unit vector $\hat{a}_{\text {life span }}$ onto the unit vectors $\hat{a}_{\mathrm{LRS}}$ and $\hat{a}_{\mathrm{RR}}$ on the D. melanogaster data (Lee et al. 2008). The scalar projection of $\vec{a}_{\text {life span }}$ measures the magnitude of the vector $\hat{a}_{\text {life span }}$ in the direction of $\hat{a}_{\mathrm{RR}}$ and $\hat{a}_{\mathrm{LRS}}$; the larger the value of the scalar projection, the greater the component of $\hat{a}_{\text {life span }}$ in the direction of $\hat{a}_{\mathrm{RR}}$ and $\hat{a}_{\mathrm{LRS}}$ and thus the lower the potential for a nutritional trade-off between these traits. The results confirmed the stronger nutritional trade-off between life span and RR, as the scalar projection between the unit vectors $\hat{a}_{\text {life span }}$ and $\hat{a}_{\mathrm{RR}}$ was 0.053 (95\% CI: 0.047-0.058), whereas the scalar projection between unit vectors $\hat{a}_{\text {life span }}$ and $\hat{a}_{\mathrm{LRS}}$ and between $\hat{a}_{\mathrm{LRS}}$ and $\hat{a}_{\mathrm{RR}}$ were 1.716 (95\% CI: $1.632-1.799)$ and 10.039 (95\% CI: 9.188-10.899), respectively. Note that these results corroborate our previous findings that the nutritional trade-off is weak between
LRS and RR (tables 1, 3), as the scalar projection between unit vectors $\hat{a}_{\text {LRS }}$ and $\hat{a}_{\mathrm{RR}}$ was high.

Finally, we investigated which nutrient was likely to have the most influence in the trade-off between life span and RR. To do this, we inspected the direction of $\vec{a}_{\text {life span }}$ and $\hat{a}_{\mathrm{RR}}$ on the Cartesian plane. While $\hat{a}_{\mathrm{RR}}$ lies in the first quadrant with positive slopes for both $P$ and $C$ intake on RR, $\vec{a}_{\text {life span }}$ is located in the second quadrant, with a negative slope of $P$ intake and a positive slope of $C$ intake on life span. This suggests that the nutritional trade-off between life span and $R R$ is driven by the opposite effects of $P$ intake on life span and reproduction.

\section{The Vector of Position Approach Can Be Used for More than Two Nutritional Dimensions}

Our approach can in theory be applied to high-dimensional data (fig. $2 F$ ). To exemplify this functionality, we simulated the intake of protein $(P)$, carbohydrates $(C)$ and fat $(F)$ on the expression of three hypothetical traits (i.e., trait $A, B$, and $\mathrm{C}$ ), so that the vectors of position $\vec{p}_{\mathrm{A}}, \vec{p}_{\mathrm{B}}$, and $\vec{p}_{\mathrm{C}}$ had coordinates (for details, see "Supplementary Methods" and fig. S1):

$$
\begin{aligned}
& \vec{p}_{\mathrm{A}}=\left(\begin{array}{lll}
P_{\mathrm{A}}^{*} & C_{\mathrm{A}}^{*} & F_{\mathrm{A}}^{*}
\end{array}\right)=\left(\begin{array}{llll}
50.2 & 30.2 & 4.9
\end{array}\right), \\
& \vec{p}_{\mathrm{B}}=\left(\begin{array}{llll}
P_{\mathrm{B}}^{*} & C_{\mathrm{B}}^{*} & F_{\mathrm{B}}^{*}
\end{array}\right)=\left(\begin{array}{llll}
50.1 & 30.2 & 5.0
\end{array}\right), \\
& \vec{p}_{\mathrm{C}}=\left(\begin{array}{llll}
P_{\mathrm{C}}^{*} & C_{\mathrm{C}}^{*} & F_{\mathrm{C}}^{*}
\end{array}\right)=\left(\begin{array}{lll}
50.0 & 70.5 & 5.3
\end{array}\right),
\end{aligned}
$$

We first compared vectors $\vec{p}_{\mathrm{A}}$ and $\vec{p}_{\mathrm{B}}$ and found an estimate of $\theta=0.206^{\circ}\left(95 \% \mathrm{CI}: 0.070^{\circ}-0.033^{\circ}\right)$. This suggests that the expression of traits $\mathrm{A}$ and $\mathrm{B}$ have similar nutritional requirements, as could be expected from the similar coordinates of the vectors $\vec{p}_{\mathrm{A}}$ and $\vec{p}_{\mathrm{B}}$. We then compared the vectors $\vec{p}_{\mathrm{A}}$ and $\vec{p}_{\mathrm{C}}$ and found that $\theta=23.32^{\circ}$ (95\% CI: $23.15^{\circ}-23.33^{\circ}$ ), indicating a stronger nutritional trade-off be-

Table 3: Comparative application of the vector of position approach

\begin{tabular}{lc}
\hline Angle, species & Estimate $(95 \% \mathrm{CI})$ \\
\hline$\alpha:$ & \\
Drosophila & $50.02053(48.22801-52.16193)$ \\
Bactrocera & $29.34615(25.38286-38.79428)$ \\
$\beta: \quad$ Drosophila & $57.76124(56.38002-59.38668)$ \\
$\quad$ Bactrocera & $67.18635(66.7606-69.50664)$ \\
$\gamma: \quad$ & $56.62615(55.7261-56.98359)$ \\
Drosophila & $72.55797(58.69577-80.35599)$ \\
\hline
\end{tabular}

Note: Shown are comparisons of the standardized performance landscapes of reproductive rate between two fruit fly species: Drosophila melanogaster (data from Lee et al. 2008) and Bactrocera tryoni (data from Fanson et al. 2009). Data are estimates (95\% credible intervals [CIs]) of the direction angles $\alpha, \beta$, and $\gamma$ for the vectors $\vec{a}_{\text {Drosophila }}$ and $\vec{a}_{\text {Bactrocera }}$. All angles are given in degrees. 
tween traits A and C than between traits A and B (see fig. S1, available online). In principle, the vector of position approach can be applied to $n$ number of nutrients. This is important because nutritional geometry studies increasingly investigate the effects of more than two nutrients on fitness traits (Gosby et al. 2014; Solon-Biet et al. 2014; Solon-Biet et al. 2015), and visual inspections of nutritional trade-offs in high-dimensional spaces can be tedious (if possible at all).

\section{The Vector of Position Approach Can Be Used for Comparing Species}

An important advance of the vector of position approach is the possibility to compare traits or trade-offs within species (e.g., males and females) but also across species. In this latter case, the angle that separates the vectors of $P$ and $C$ intake is not sufficient because we are now interested in both nutrient intake as well as the expression level of the trait between species. For simplicity, let us consider two-dimensional vectors of $P$ and $C$ and this time include the third dimension to the vectors that describe the expression level of the trait (fig. $3 A$ ). This step is needed because the trait can be maximized in the same location in the nutrient space but with different expression levels (i.e., the difference may be on the $Z$-axis). Consider the vector of position $\vec{p}_{\mathrm{sp} i}$ as in equation (6). To work with different species, it is necessary to standardize the coordinates of the vector so that the vectors $\vec{p}_{i}$ are now defined as

$$
\begin{aligned}
& \overrightarrow{\hat{p}}_{\mathrm{sp} 1}=\left(\begin{array}{lll}
\hat{P}_{\mathrm{sp} 1}^{*} & \hat{C}_{\mathrm{sp} 1}^{*} & \hat{w}_{\mathrm{sp} 1}^{*}
\end{array}\right), \\
& \overrightarrow{\hat{p}}_{\mathrm{sp} 2}=\left(\begin{array}{lll}
\hat{P}_{\mathrm{sp} 2}^{*} & \hat{C}_{\mathrm{sp} 2}^{*} & \hat{w}_{\mathrm{sp} 2}^{*}
\end{array}\right),
\end{aligned}
$$

where

$$
\begin{gathered}
\hat{P}_{\mathrm{sp} 1}=\frac{P}{\bar{P}}, \\
\hat{C}_{\mathrm{sp} 1}=\frac{C}{\bar{C}}, \\
\hat{w}_{\mathrm{sp} 1}=\frac{w}{\bar{w}} .
\end{gathered}
$$

The terms $\hat{P}, \hat{C}$, and $\hat{w}$ are the standardized values of the protein $(P)$ and carbohydrate $(C)$ intake and the trait of interest $(w)$. Having defined the standardized position vectors $\overrightarrow{\hat{p}}_{\mathrm{sp} 1}$ and $\overrightarrow{\hat{p}}_{\mathrm{sp} 2}$, we can use the vector of position approach to calculate the angle $\theta$ that separates these two vectors. Here $\theta$ also includes the differences in the expression of traits (on the $Z$-axis), which in biological terms can be interpreted as the difference in the maximum standardized expression of the trait of interest when species are at the peak in the performance landscape. It is also possible to calculate the directional angles $\alpha, \beta$, and $\gamma$ separating the vector and the $X$-,
$Y$-, and $Z$-axes, respectively, which provides information regarding the differences in standardized $P$ and $C$ intake as well as trait expression between species (fig. $3 B$ ). The directional angles $\alpha, \beta$, and $\gamma$ can be calculated as follows:

$$
\begin{aligned}
& \alpha=\cos ^{-1}\left(\frac{\hat{P}_{\mathrm{sp} 1}^{*}}{\sqrt{\hat{P}_{\mathrm{sp}}^{* 2}+\hat{C}_{\mathrm{sp}}^{* 2}+\hat{w}_{\mathrm{sp}}^{* 2}}}\right), \\
& \beta=\cos ^{-1}\left(\frac{\hat{C}_{\mathrm{sp} 1}^{*}}{\sqrt{\hat{P}_{\mathrm{sp}}^{* 2}+\hat{C}_{\mathrm{sp}}^{* 2}+\hat{w}_{\mathrm{sp}}^{* 2}}}\right), \\
& \gamma=\cos ^{-1}\left(\frac{\hat{w}_{\mathrm{sp} 1}^{*}}{\sqrt{\hat{P}_{\mathrm{sp}}^{* 2}+\hat{C}_{\mathrm{sp}}^{* 2}+\hat{\mathcal{W}}_{\mathrm{sp}}^{* 2}}}\right) .
\end{aligned}
$$

If the peaks are located in the same region of the standardized performance landscapes, the angles $\alpha$ and $\beta$ are likely to be similar between species 1 and 2 , as the vectors $\overrightarrow{\hat{p}}_{\text {sp } i}$ point in the same direction in space (fig. $3 B$ ). This property could be used to infer the mechanisms underlying the speciesspecific effects of nutrition on trait expression. For example, consider the $X$-axis as being $P$ intake. If the angles $\alpha_{\text {sp } 1}$ and $\alpha_{\mathrm{sp} 2}$ measure the distance between vectors and the $X$-axis, the angles $\alpha_{\mathrm{sp} 1}$ and $\alpha_{\mathrm{sp} 2}$ indicate how strongly the standardized optimal $P$ intake influences the expression of traits for each species. If the difference between $\alpha_{\mathrm{sp} 1}$ and $\alpha_{\mathrm{sp} 2}$ is large, the optimal standardized $P$ intake between species is located far from each other, and thus $P$ intake is likely a key modulator of the differences in trait expression between the species.

To illustrate this approach, we compared the nutritional requirements for the RR of two fruit fly species: $D$. melanogaster (Lee et al. 2008) and Bactrocera tryoni (Fanson et al. 2009; fig. 3C-3E). We standardized the performance landscapes of the two species according to equation (13). Using the previously described machine learning algorithm (see "Data and Statistical Analyses"), we estimated the region of the standardized peak in RR in the standardized performance landscape for both species (fig. $3 C-3 E$ ) and used these values as the coordinates of the standardized vectors of position $\vec{p}_{\text {Drosophila }}$ and $\vec{p}_{\text {Bactrocera }}$ according to equation (12). We then calculated the average, the minimum, and the maximum values of the $\theta$ angle separating $\vec{p}_{\text {Drosophila }}$ and $\vec{p}_{\text {Bactrocera }}$. On average, we found that $\theta=21.28^{\circ}$, with a minimum value of $11.30^{\circ}$ and a maximum value of $29.88^{\circ}$, suggesting a relatively large degree of separation between the expression of the trait between the two species.

We then investigated this separation in terms of $P$ and $C$ intake and expression level of the trait by calculating the angles $\alpha, \beta$, and $\gamma$ between the vectors $\vec{p}_{\text {Drosophila }}$ and $\vec{p}_{\text {Bactrocera }}$ and the standardized $X$-, $Y$-, and $Z$-axes, respectively (table 3 ). We 


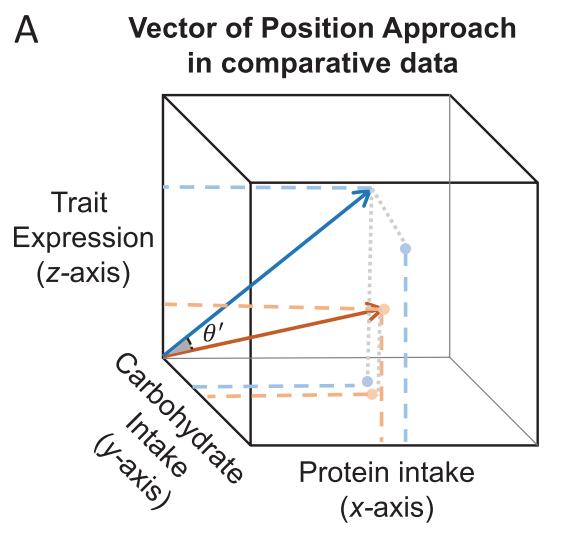

C

Reproductive rate (RR)

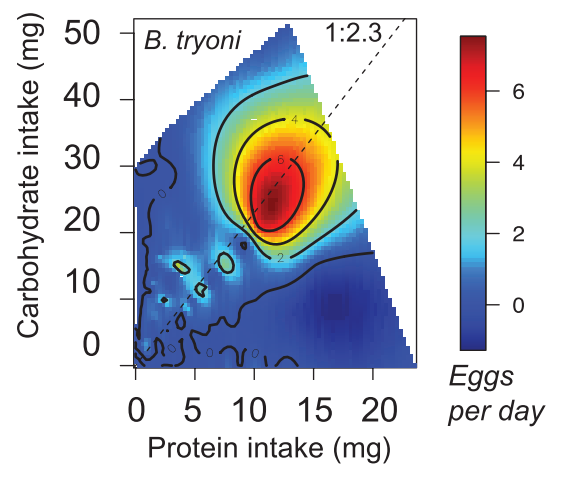

$E$

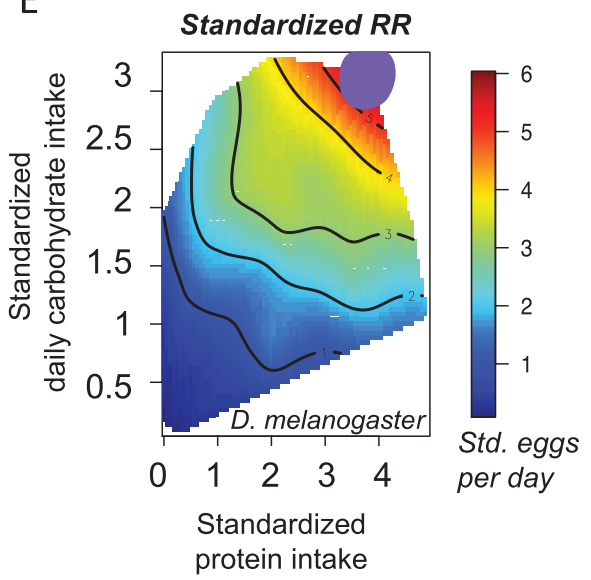

B
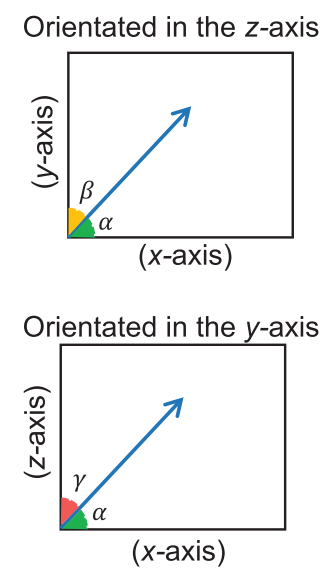

D

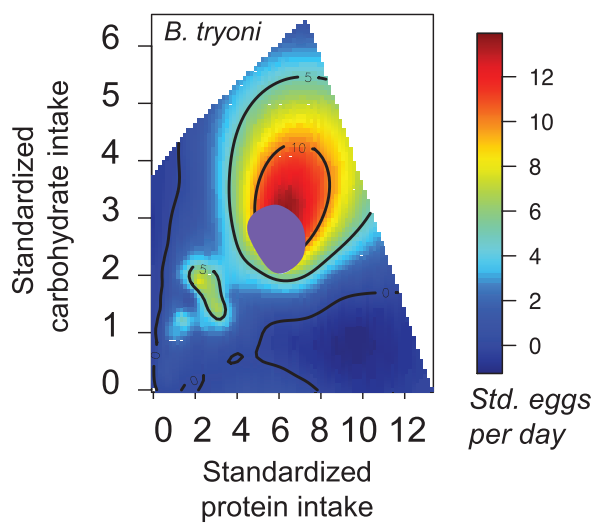

Figure 3: Between-species comparison. $A$, Schematic representation of a three-dimensional vector in a performance landscape of protein, carbohydrate, and trait expression. $B$, Directional angles $\alpha, \beta$, and $\gamma$. Top, figure orientated according to the $Z$-axis (perpendicular to the plane of the paper); bottom, figure orientated according to the $Y$-axis (perpendicular to the plane of the paper). $C$, Performance landscapes of reproductive rate (RR) of Bactrocera tryoni females (data from Fanson et al. 2009). The dashed line highlights the protein-to-carbohydrate ratio that maximizes the trait. $D, E$, Mean-standardized performance landscapes of the RR of $B$. tryoni (data from Fanson et al. 2009) and Drosophila melanogaster (data from Lee et al. 2008), respectively. Purple highlights the region estimated as the peak in the performance landscape from the support-vector machine learning model. Data for the performance landscapes are available in the Dryad Digital Repository (https://dx.doi.org/10.5061/dryad.tp7519s; Morimoto and Lihoreau 2019). 
found a marked difference in the angles $\alpha$ and $\gamma\left(\sim 21^{\circ}\right.$ and $16^{\circ}$, respectively), whereas there was only a small difference for the angle $\beta\left(\sim 9^{\circ}\right)$, suggesting that the difference in nutritional requirements for the $R R$ of females of the two species are mainly driven by differences in species-specific standardized $P$ intake and expression level of the trait.

\section{Discussion}

We build on recent analytical advances to introduce a generalized vector-based approach to quantify the strength of nutritional trade-offs in nutritional geometry data and compare them across studies. The vector of position approach strictly considers vectors of positions in the real positive region of the nutrient space, meaning that the approach calculates the true separation between key areas in the nutrient spaces (peaks and valleys) and hence the true strength of nutritional tradeoffs. This facilitates quantitative analyses of nutritional tradeoffs in a broad range of studies, including complex experimental designs considering multiple nutritional dimensions, and allows for the accurate inferences of the forces driving the nutritional trade-off in expression of life-history traits within and between species.

A major advantage of our approach in comparison with previous methods is that it is not constrained to two dimensions. Such generalization is key when dealing with three or more nutrients (or any other relevant food components) and in which data visualization can be difficult. Although initially developed for analyses with two nutrients (e.g., typically the macronutrients carbohydrates and protein; Simpson and Raubenheimer 1993), nutritional geometry is increasingly used for exploring animal performances in high-dimension nutrient spaces, as illustrated by recent data on the importance of carbohydrates, protein, and fat in reproduction, life span, and other aspects of animal health (Hewson-Hughes et al. 2011, 2012; Gosby et al. 2014; Solon-Biet et al. 2014, 2015). There is also growing interest in investigating the effects of other food components, such as individual fatty acids (Arien et al. 2015), mineral salts (Simpson et al. 2006), water (Fanson et al. 2012), or even individual amino acids (e.g., Grandison et al. 2009; Arganda et al. 2017; Piper et al. 2017), which may soon require analyses in nutrient spaces with as many as 22 dimensions, for which visual inspections of nutrient spaces or inaccurate estimates of distance between performance peaks will not suffice. Our approach therefore provides a unique solution to quantify these complex nutritional trade-offs.

The vector of position approach also allows for quantitative explorations of global patterns in the performance landscapes that can yield important, yet often ignored, information about how nutritional trade-offs are resolved within and across life stages. For instance, in drosophilids nutritional geometry studies show that both larval and adult diets modulate reproduction and other fitness-related traits in adulthood (e.g., Lee et al. 2008; Reddiex et al. 2013; Jensen et al. 2015; Matavelli et al. 2015; Morimoto and Wigby 2016; de Carvalho and Mirth 2017; Silva-Soares et al. 2017). The vector of position approach allows for the comparison of the performance landscapes in groups where the larvae, adult, or both are fed different diets, providing a powerful quantitative framework for understanding the effects of nutrition on an organism's ontogeny.

Another major advance of our approach is the possibility of comparing performance landscapes across species. Comparative studies of nutritional traits can yield many fundamental insights into the role of nutrition in the evolution of physiological and behavioral strategies and ecological processes across species (Behmer and Joern 2008). For instance, primate comparative research shows that nutrient availability in ancestral diets has shaped appetite and food selection that vary greatly among species (Felton et al. 2009; Rothman et al. 2011; Johnson et al. 2013), providing key insights for our understanding of the current human obesity epidemic (Simpson and Raubenheimer 2014). By reanalyzing two published data sets in fruit flies using the vector of position approach, we quantified species-specific differences in female RR and identified that this difference is driven by differences in species-specific standardized protein intake and expression level of the trait. A potential explanation for this result is that Lee et al. (2008) used mated female Drosophila melanogaster that require increased protein intake for egg production (Yapici et al. 2008; Ribeiro and Dickson 2010; Gligorov et al. 2013; Perry et al. 2013; Walker et al. 2015), while Fanson et al. (2009) used unmated female Bactrocera tryoni; thus, future studies using standardized protocols are called for. In future research, accurate quantification and comparisons of performance landscapes in species exhibiting different social lifestyles will help investigate the role of nutrition in social evolution (Lihoreau et al. 2015, 2017), for instance, to explain social transitions from solitary to gregarious stages (Simpson et al. 2006). Comparative analyses can also reveal key adaptations to past and present ecological conditions that underpin species niche occupation and determine the responses to new nutritional conditions that arise through environmental changes. In this context, the vector of position approach could be implemented to attain a more detailed characterization and comparison of performance landscapes across social species or species occupying different ecological niches. This could allow, for example, testing of previous models suggesting that specialist species are likely to have a sharp peak in the performance landscape surrounded by valleys due to low tolerance for diet diversity, whereas generalist species are likely to have a broader peak in the performance landscape with relatively few valleys due to high tolerance for diet diversity (Simpson et al. 2004; Behmer 2009). 
More generally, our analytical approach can help study how complex nutritional processes interact across levels of biological organization. The vector of position approach is highly flexible and can facilitate comparisons of the performance landscapes of a wide range of interacting agents beyond just conspecifics or competitors, such as host and parasites as well as hosts and microbiota. This is important because nutrition is a key factor modulating the stability of many of these interspecific associations (Ponton et al. 2011; Wong et al. 2015), which are known to have major influences on the survival and fitness of individuals (e.g., Coyte et al. 2015; Rosshart et al. 2017; Keebaugh et al. 2018; and references therein). Thus, implementing the vector of position approach in quantitative analyses of the performance landscapes of the microbiota or pathogen and the host can help identify the nutritional conditions in which stability is achieved and potential evolutionary conflicts are resolved, hence favoring the emergence and maintenance of interspecific associations (e.g., Shik et al. 2016). All of these comparisons are currently made by eye or are statistically compared in two-dimensional spaces, and therefore quantitative approaches such as the one proposed here yield considerable promise for understanding the role of nutrition in the evolution of animal physiology, behavior, and ecology.

Data availability. All data sets are available in the Dryad Digital Repository (https://dx.doi.org/10.5061/dryad.tp7519s; Morimoto and Lihoreau 2019). All code is provided as supplemental material.

\section{Acknowledgments}

We are grateful to Fleur Ponton, Russell Bonduriansky, and Emilie Snell-Rood as well as two anonymous reviewers for their constructive comments on the manuscript. We are grateful to Ben Fanson, Kwang Lee, and Phil Taylor for sharing their data. This research was funded by a grant from the Agence Nationale de la Recherche to M.L. (ANR-16-CE02-0002-01).

\section{Literature Cited}

Arganda, S., S. Bouchebti, S. Bazazi, S. Le Hesran, C. Puga, G. Latil, S. J. Simpson, and A. Dussutour. 2017. Parsing the life-shortening effects of dietary protein: effects of individual amino acids. Proceedings of the Roval Societv B 284:20162052.

Arien, Y., A. Dag, S. Zarchin, T. Masci, and S. Shafir. 2015. Omega-3 deficiency impairs honey bee learning. Proceedings of the National Academv of Sciences of the USA 112:15761-15766.

Behmer, S. T. 2009. Insect herbivore nutrient regulation. Annual Review of Entomology 54:165-187.

Behmer, S. T., and A. Joern. 2008. Coexisting generalist herbivores occupy unique nutritional feeding niches. Proceedings of the $\mathrm{Na}$ tional Academv of Sciences of the USA 105:1977-1982.

Bunning, H., L. Bassett, C. Clowser, J. Rapkin, K. Jensen, C. M. House, C. R. Archer, and J. Hunt. 2016. Dietary choice for a balanced nu- trient intake increases the mean and reduces the variance in the reproductive performance of male and female cockroaches. Ecology and Evolution 6:4711-4730.

Bunning, H., J. Rapkin, L. Belcher, C. R. Archer, K. Jensen, and J. Hunt. 2015. Protein and carbohydrate intake influence sperm number and fertility in male cockroaches, but not sperm viability. Proceedings of the Roval Societv B 282:20142144.

Cotter, S. C., S. J. Simpson, D. Raubenheimer, and K. Wilson. 2011. Macronutrient balance mediates trade-offs between immune function and life history traits. Functional Ecology 25:186-198.

Coyte, K. Z., J. Schluter, and K. R. Foster. 2015. The ecology of the microbiome: networks, competition, and stability. Science 350:663666.

de Carvalho, M. J. A., and C. K. Mirth. 2017. Food intake and food choice are altered by the developmental transition at critical weight in Drosophila melanogaster. Animal Behaviour 126:195-208.

del Castillo, E., J. Hunt, and J. Rapkin. 2016. OptimaRegion: confidence regions for Optima. R package version 0.2.

Fanson, B. G., C. W. Weldon, D. Pérez-Staples, S. J. Simpson, and P. W. Taylor. 2009. Nutrients, not caloric restriction, extend lifespan in Queensland fruit flies (Bactrocera tryoni). Aging Cell 8:514523.

Fanson, B. G., S. Yap, and P. W. Taylor. 2012. Geometry of compensatory feeding and water consumption in Drosophila melanogaster. Lournal of Experimental Biology 215:766-773.

Felton, A. M., A. Felton, D. Raubenheimer, S. J. Simpson, W. J. Foley, J. T. Wood, I. R. Wallis, and D. B. Lindenmayer. 2009. Protein content of diets dictates the daily energy intake of a free-ranging primate. Behavioral Ecology 20:685-690.

Gligorov, D., J. L. Sitnik, R. K. Maeda, M. F. Wolfner, and F. Karch. 2013. A novel function for the Hox gene Abd-B in the male accessory gland regulates the long-term female post-mating response in Drosophila. PLoS Genetics 9:e1003395.

Gosby, A. K., A. D. Conigrave, D. Raubenheimer, and S. J. Simpson. 2014. Protein leverage and energy intake. Obesitv Reviews 15:183191.

Grandison, R. C., M. D. W. Piper, and L. Partridge. 2009. Amino-acid imbalance explains extension of lifespan by dietary restriction in Drosophila. Nature 462:1061-1064.

Hadfield, J. D. 2010. MCMC methods for multi-response generalized linear mixed models: the MCMCglmm R package. Iournal of Statistical Software 33:1-22.

Heath, T. L. 1956. The thirteen books of Euclid's elements. Encyclopædia Britannica, Chicago.

Hewson-Hughes, A. K., V. L. Hewson-Hughes, A. Colyer, A. T. Miller, S. J. McGrane, S. R. Hall, R. F. Butterwick, S. J. Simpson, and D. Raubenheimer. 2012. Geometric analysis of macronutrient selection in breeds of the domestic dog, Canis lupus familiaris. Behavioral Ecology 24:293-304.

Hewson-Hughes, A. K., V. L. Hewson-Hughes, A. T. Miller, S. R. Hall, S. J. Simpson, and D. Raubenheimer. 2011. Geometric analysis of macronutrient selection in the adult domestic cat, Felis catus. Iournal of Experimental Biology 214:1039-1051.

House, C. M., K. Jensen, J. Rapkin, S. Lane, K. Okada, D. J. Hosken, and J. Hunt. 2016. Macronutrient balance mediates the growth of sexually selected weapons but not genitalia in male broad-horned beetles. Functional Ecology 30:769-779.

Hunt, J., R. Brooks, M. D. Jennions, M. J. Smith, C. L. Bentsen, and L. F. Bussiere. 2004. High-quality male field crickets invest heavily in sexual display but die young. Nature 432:1024-1027. 
Jensen, K., C. McClure, N. K. Priest, and J. Hunt. 2015. Sex-specific effects of protein and carbohydrate intake on reproduction but not lifespan in Drosophila melanogaster. Aging Cell 14:605-615.

Johnson, C. A., D. Raubenheimer, J. M. Rothman, D. Clarke, and L. Swedell. 2013. 30 days in the life: daily nutrient balancing in a wild chacma baboon. PLoS ONE 8:e70383.

Keebaugh, E. S., R. Yamada, B. Obadia, W. B. Ludington, and W. W. Ja. 2018. Microbial quantity impacts Drosophila nutrition, development, and lifespan. iScience 4:247-259.

Kuhn, M. 2017. Caret: classification and regression training. R package version 6.0-78.

Lee, K. P., S. J. Simpson, F. J. Clissold, R. Brooks, J. W. Ballard, P. W. Taylor, N. Soran, and D. Raubenheimer. 2008. Lifespan and reproduction in Drosophila: new insights from nutritional geometry. Proceedings of the National Academy of Sciences of the USA 105:2498-2503.

Lihoreau, M., J. Buhl, M. A. Charleston, G. A. Sword, D. Raubenheimer, and S. J. Simpson. 2015. Nutritional ecology beyond the individual: a conceptual framework for integrating nutrition and social interactions. Ecology Letters 18:273-286.

Lihoreau, M., M. A. Charleston, A. M. Senior, F. J. Clissold, D. Raubenheimer, S. J. Simpson, and J. Buhl. 2017. Collective foraging in spatially complex nutritional environments. Philosophical Transactions of the Roval Society B 372:20160238.

Maklakov, A. A., S. J. Simpson, F. Zajitschek, M. D. Hall, J. Dessmann, F. Clissold, D. Raubenheimer, R. Bonduriansky, and R. C. Brooks. 2008. Sex-specific fitness effects of nutrient intake on reproduction and lifespan. Current Biology 18:1062-1066.

Matavelli, C., M. J. Carvalho, N. E. Martins, and C. K. Mirth. 2015. Differences in larval nutritional requirements and female oviposition preference reflect the order of fruit colonization of Zaprionus indianus and Drosophila simulans. Journal of Insect Physiology 82:66-74.

Morimoto, J., and M. Lihoreau. 2019. Data from: Quantifying nutritional trade-offs across multidimensional performance landscapes. American Naturalist, Dryad Digital Repository, https://dx.doi.org /10.5061/dryad.tp7519s.

Morimoto, J., and S. Wigby. 2016. Differential effects of male nutrient balance on pre- and post-copulatory traits, and consequences for female reproduction in Drosophila melanogaster. Scientific Reports 6:27673.

Nychka, D., R. Furrer, J. Paige, and S. Sain. 2017. fields: tools for spatial data. R package version 9.6.

Perry, J. C., L. Sirot, and S. Wigby. 2013. The seminal symphony: how to compose an ejaculate. Trends in Ecology and Evolution 28:414422.

Piper, M. D., G. A. Soultoukis, E. Blanc, A. Mesaros, S. L. Herbert, P. Juricic, X. He, et al. 2017. Matching dietary amino acid balance to the in silico-translated exome optimizes growth and reproduction without cost to lifespan. Cell Metabolism 25:610-621.

Ponton, F., K. Wilson, S. C. Cotter, D. Raubenheimer, and S. J. Simpson. 2011. Nutritional immunology: a multi-dimensional approach. PLoS Pathogens 7:e1002223.

Ponton, F., K. Wilson, A. Holmes, D. Raubenheimer, K. L. Robinson, and S. J. Simpson. 2015. Macronutrients mediate the functional relationship between Drosophila and Wolbachia. Proceedings of the Roval Society B 282:20142029.

Rapkin, J., K. Jensen, C. R. Archer, C. M. House, S. K. Sakaluk, E. D. Castillo, and J. Hunt. 2018. The geometry of nutrient space-based life-history trade-offs: sex-specific effects of macronutrient intake on the trade-off between encapsulation ability and reproductive effort in decorated crickets. American Naturalist 191:452-474.

Rapkin, J., K. Jensen, S. M. Lane, C. M. House, S. K. Sakaluk, and J. Hunt. 2016. Macronutrient intake regulates sexual conflict in decorated crickets. Journal of Evolutionary Biology 29:395-406.

Raubenheimer, D., and S. Simpson. 1993. The geometry of compensatory feeding in the locust. Animal Behaviour 45:953-964.

2018. Nutritional ecology and foraging theory. Current Opinion in Insect Science 27:38-45.

R Development Core Team. 2017. R: a language and environment for statistical computing. R Foundation for Statistical Computing, Vienna. http://www.R-project.org/.

Reddiex, A. J., T. P. Gosden, R. Bonduriansky, and S. F. Chenoweth. 2013. Sex-specific fitness consequences of nutrient intake and the evolvability of diet preferences. American Naturalist 182:91102.

Ribeiro, C., and B. J. Dickson. 2010. Sex peptide receptor and neuronal TOR/S6K signaling modulate nutrient balancing in Drosophila. Current Biology 20:1000-1005.

Roff, D. A. 2002. Life history evolution. Vol. 7. Sinauer, Sunderland, MA.

Rosshart, S. P., B. G. Vassallo, D. Angeletti, D. S. Hutchinson, A. P. Morgan, K. Takeda, H. D. Hickman, et al. 2017. Wild mouse gut microbiota promotes host fitness and improves disease resistance. Cell 171:1015-1028.

Rothman, J. M., D. Raubenheimer, and C. A. Chapman. 2011. Nutritional geometry: gorillas prioritize non-protein energy while consuming surplus protein. Biology Letters 7:847-849.

Schwenke, R. A., B. P. Lazzaro, and M. F. Wolfner. 2016. Reproductionimmunity trade-offs in insects. Annual Review of Entomology 61:239256.

Semaniuk, U., K. Feden'ko, I. S. Yurkevych, K. B. Storey, S. J. Simpson, and O. Lushchak. 2018. Within-diet variation in rates of macronutrient consumption and reproduction does not accompany changes in lifespan in Drosophila melanogaster. Entomologia Experimentalis et Applicata 166:74-80.

Shik, J. Z., E. B. Gomez, P. W. Kooij, J. C. Santos, W. T. Wcislo, and J. J. Boomsma. 2016. Nutrition mediates the expression of cultivarfarmer conflict in a fungus-growing ant. Proceedings of the National Academy of Sciences of the USA 113:10121-10126.

Silva-Soares, N. F., A. Nogueira-Alves, P. Beldade, and C. K. Mirth. 2017. Adaptation to new nutritional environments: larval performance, foraging decisions, and adult oviposition choices in Drosophila suzukii. BMC Ecology 17:21.

Simpson, S. J., D. G. Le Couteur, D. E. James, J. George, J. E. Gunton, S. M. Solon-Biet, and D. Raubenheimer. 2017a. The geometric framework for nutrition as a tool in precision medicine. Nutrition and Healthy Aging 4:217-226.

Simpson, S. J., and D. Raubenheimer. 1993. A multi-level analysis of feeding behaviour: the geometry of nutritional decisions. Philosophical Transactions of the Roval Society B 342:381-402.

. 2012. The nature of nutrition: a unifying framework from animal adaptation to human obesity. Princeton University Press, Princeton, NJ.

- 2014. Perspective: tricks of the trade. Nature 508:S66.

Simpson, S. J., D. Raubenheimer, M. A. Charleston, and F. J. Clissold. 2010. Modelling nutritional interactions: from individuals to communities. Trends in Ecology and Evolution 25:53-60.

Simpson, S. J., D. Raubenheimer, V. C. Cogger, L. Macia, S. M. SolonBiet, D. G. Le Couteur, and J. George. 2017b. The nutritional ge- 
ometry of liver disease including non-alcoholic fatty liver disease (NAFLD). Journal of Hepatology 68:316-325.

Simpson, S. J., R. M. Sibly, K. P. Lee, S. T. Behmer, and D. Raubenheimer. 2004. Optimal foraging when regulating intake of multiple nutrients. Animal Behaviour 68:1299-1311.

Simpson, S. J., G. A. Sword, P. D. Lorch, and I. D. Couzin. 2006. Cannibal crickets on a forced march for protein and salt. Proceedings of the National Academv of Sciences of the USA 103:4152-4156.

Solon-Biet, S. M., A. C. McMahon, J. W. O. Ballard, K. Ruohonen, L. E. Wu, V. C. Cogger, A. Warren, et al. 2014. The ratio of macronutrients, not caloric intake, dictates cardiometabolic health, aging, and longevity in ad libitum-fed mice. Cell Metabolism 19:418430.

Solon-Biet, S. M., K. A. Walters, U. K. Simanainen, A. C. McMahon, K. Ruohonen, J. W. O. Ballard, D. Raubenheimer, D. J. Handelsman, D. G. Le Couteur, and S. J. Simpson. 2015. Macronutrient balance, reproductive function, and lifespan in aging mice. Proceedings of the National Academv of Sciences of the USA 112:3481-3486.

Stearns, S. C. 1989. Trade-offs in life-history evolution. Functional Ecology 3:259-268.
Trumper, S., and S. Simpson. 1993. Regulation of salt intake by nymphs of Locusta migratoria. Journal of Insect Physiology 39:857-864.

Walker, S. J., V. M. Corrales-Carvajal, and C. Ribeiro. 2015. Postmating circuitry modulates salt taste processing to increase reproductive output in Drosophila. Current Biology 25:2621-2630.

Wong, A. C.-N., A. Holmes, F. Ponton, M. Lihoreau, K. Wilson, D. Raubenheimer, and S. J. Simpson. 2015. Behavioral microbiomics: a multi-dimensional approach to microbial influence on behavior. Frontiers in Microbiology 6:1359.

Yapici, N., Y. J. Kim, C. Ribeiro, and B. J. Dickson. 2008. A receptor that mediates the post-mating switch in Drosophila reproductive behaviour. Nature 451:33-37.

\section{References Cited Only in the Supplemental Material}

Soetaert, K. 2017. plot3D: plotting multi-dimensional data. R package version 1.1.1.

Associate Editor: Emilie C. Snell-Rood Editor: Russell Bonduriansky

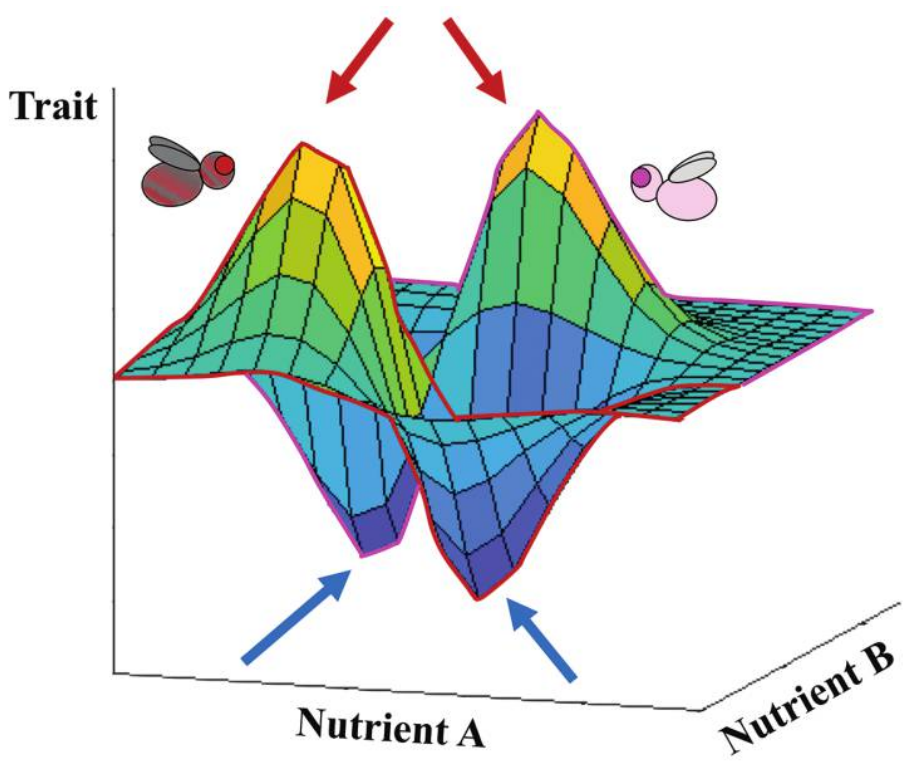

The Morimoto and Lihoreau method allows for the comparison of diets that maximize (red arrows) and minimize (blue arrows) the performance of multiple species. The figure shows two performance landscapes of two hypothetical fly species (pink and magenta). Credit: Juliano Morimoto. 